\title{
Cross Validation of Doppler Ultrasound and Grayscale Imaging for Discrimination of Benign and Malignant Adnexal Masses
}

\author{
Syeda Batool Mazhar, FRCOG, MRCOG, MBBS;1 and Kausar Jabeen, FCPS, MBBS2 \\ 1 Unit II, Mother and Child Health Center \\ Pakistan Institute of Medical Sciences \\ Islamabad, Pakistan \\ 2Specialist Obstetrics and Gynecology, Prince Abdul Mohsin General Hospital \\ Al Ula Madina Munawara Region, Kingdom of Saudi Arabia
}

\begin{abstract}
:
Objective: To prospectively assess the diagnostic performance of Doppler ultrasound and grayscale imaging for the discrimination of benign and malignant adnexal masses.

Study design: Observational study.

Duration: January 2002 to December 2003.

Setting: Mother and Child Health (MCH) Center, Unit II, Pakistan Institute of Medical Sciences, Islamabad, Pakistan.

Subjects: Patients with an adnexal mass who were scheduled for surgery in the study. Patients managed conservatively were excluded.

Methods: All subjects underwent a preoperative grayscale and color Doppler ultrasound. On the basis of subjective evaluation, each tumor was classified as benign or malignant. Results of diagnostic studies were correlated with pathological evaluations of the excised specimens.

Main outcome measures: Sensitivity, specificity, positive predictive value, and negative predictive value of both modes of ultrasound.

Results: A total of 117 patients were examined during the study period. Grayscale echopatterns considered ominous for malignancy were solid components, thick septae, papillary projections, and ascites. Using Doppler flow studies, malignant neoplasms offered lower resistance to blood flow, as measured by the resistance index (RI) and the pulsatility index (PI). The mean PI of benign tumors was $1.6 \pm 0.4$ vs. $0.7 \pm 0.1$ of malignant masses, and the mean RI was $1.2 \pm 0.3$ vs. $0.4 \pm 0.1$ of malignant tumors. The sensitivity and specificity of grayscale was $82 \%$ and $80 \%$, while for Doppler imaging it was $91 \%$ and $95 \%$, respectively. The positive predictive value of grayscale was $30.0 \%$ vs. a $66.7 \%$ predictive value of Doppler imaging, and the negative predictive value was $98 \%$ vs. $99 \%$ of Doppler ultrasound.

Conclusion: Doppler ultrasound offers a higher sensitivity and specificity for differentiation of adnexal masses compared to grayscale ultrasound.
\end{abstract}

Key words: Benign adnexal masses, malignant adnexal masses, Doppler ultrasound, grayscale ultrasound. 


\section{Introduction}

A dnexal masses are one of the most frequent reasons for referral to a gynecologist because they could be malignant. ${ }^{1}$ Four to $24 \%$ of masses discovered before menopause and between $39 \%$ and $63 \%$ of those found after menopause are malignant. ${ }^{2-4}$ Although ovarian cancer represents only $5 \%$ of all cancers, it is the fourth leading cause of death from cancer among women and the most fatal among gynecologic tumors. ${ }^{5}$ Despite advances in treatment, ovarian cancer has consistently had the highest case fatality ratio of all gynecologic malignancies, with a 5-year survival rate of $40-50 \%$ for all stages. ${ }^{6-8}$ There has been little improvement in survival over the past 20-30 years. ${ }^{9}$ While it is possible that a treatment breakthrough may be made, early diagnosis of ovarian cancer is probably the best approach to achieve a reduction in morbidity and mortality.

As early malignant lesions include the formation of a complex cystic mass, most efforts point toward detection and elimination of adnexal masses. The standard evaluation for adnexal masses includes history, examination, tumor markers, and ultrasound. Whether ultrasound can differentiate between benign and malignant adnexal masses has been the subject of many studies. ${ }^{10-12}$ Grayscale ultrasound of adnexal masses using transabdominal or transvaginal routes enables accurate evaluation of the characteristics of masses. However, because of lower sensitivity, it is not possible to predict with certainty whether a mass is benign or malignant.

The role of color Doppler ultrasound in differentiating adnexal masses has been suggested in various published studies reporting diagnostic accuracies of 90\% and higher. ${ }^{13-20}$ Color Doppler studies in a developing country such as Pakistan are limited because they involve expensive equipment. This study, conducted at a tertiary care public sector hospital with use of color ultrasound, was designed to compare the diagnostic performance of grayscale and color Doppler ultrasound, and then correlate results with the histopathology report.

Correspondence should be directed by email to

Dr. Kausar Jabeen, FCPS, MBBS

drkausarniazi@yahoo.com

\section{Subjects and Methods}

This study was conducted at the Department of Gynecology and Obstetrics, Unit II, Mother and Child Health (MCH) Center, Pakistan Institute of Medical Sciences (PIMS), Islamabad, from January 2002 to December 2003. The center is a postgraduate teaching institution that provides tertiary-level care. One hundred and seventeen patients admitted to the gynecology ward at Unit II, MCH Center, PIMS, with a clinical or sonographic diagnosis of an adnexal mass (irrespective of their age and menstrual status), and scheduled for surgery were included in the study.

All the subjects were scanned with B-mode (grayscale) ultrasonography initially, using $3.5-\mathrm{MHz}$ and $5-\mathrm{MHz}$ probes for transabdominal and transvaginal scans, respectively, to assess the gross architecture of the pelvic mass and the presence of ascites. The following characteristics of the adnexal masses were recorded: size, site, septations, wall thickness, loculations, papillary projections, echogenecity (cystic, solid, mixed), and liquid phase. The cutoff value for thickness of the tumor wall and intracystic septa in benign tumors was $<5 \mathrm{~mm}$. Echopatterns considered ominous for malignancy were bilateral masses, solid components, multiloculations, irregular borders, and the presence of ascites. Simple cysts, smooth borders, clear cysts, or low-level echoes were considered more in favor of the benign nature of a tumor. On the basis of this subjective evaluation, each tumor was classified as benign or malignant. If possible, a specific diagnosis was made, e.g. "endometriosis" or "dermoid cyst." "Malignancy" was not considered a specific diagnosis.

Following grayscale ultrasonography, color flow imaging was done to determine the pattern of tumor vascularity and, finally, pulsed Doppler was superimposed. Both Doppler and grayscale scans were performed by the same physician. Scanning was performed using an Aloka SSD-1700 ultrasound system, equipped with a color and pulsed Doppler and a 3.5MHz transabdominal and 5-MHz transvaginal transducer. Using color flow imaging, the areas of intratumoral vascularization were searched. If intratumoral vessels were not visualized, color flow imaging readings were obtained from peripheral vessels. Arrangement of the vessels was noted. Thereafter, the pulsed Doppler was superimposed. When an arterial flow waveform was detected, the image was

JIMA: Volume 40, 2008 - Page 70 
frozen. From the given inputs, the resistance index (RI) and pulsatility index (PI) were calculated automatically. Resistance index is the maximum systolic velocity minus the maximum diastolic velocity, divided by peak systolic velocity. Pulsatility index is the maximum systolic velocity minus the peak diastolic velocity, divided by the mean velocity. Three separate vessels detected inside the tumor wall determined neovascularization. The final RI and PI were the lowest calculated measurement from the three assessments made at separate sites within the tumor. Different cutoff values for RI and PI to predict malignancy were reported. ${ }^{21-5}$ In this study, the cutoff value for RI was $<0.50$, and the cutoff value for PI was $<1.0$.

Sensitivity, specificity, and positive predictive and negative predictive values were calculated for both modes. All the patients included in the study underwent surgery. Subjects with clinically evident ovarian cancer had staging laparotomy. The decision regarding the surgical approach in patients with expected benign tumors, i.e. laparoscopy or laparotomy, was left to the surgeon. Results of the ultrasound studies were correlated with the operative findings and pathological evaluation of the excised specimen.

Data were collected on a predesigned performa, entered in Microsoft Excel, and analyzed on the SPSS statistical package, Version 10. The chi square test was used to determine differences, and two-by-two table was used to calculate sensitivity, specificity, positive, and negative predictive values. Statistical significance was defined as $\mathrm{P}<0.05$.

\section{Results}

One hundred and seventeen patients were examined over a 2-year period. Adnexal masses were more frequent in women $20-40$ years of age (54.7\%), compared to $28 \%$ among women older than 40 years of age. There were no patients younger than 10 years of age. Fourteen patients were postmenopausal (age range 40-70 years). The most common presenting complaints were pelvic-abdominal pain (47.9\%), infertility (21.0\%), and a mass in the abdomen (17.9\%). Among the study subjects, 106 (90.5\%) had benign ovarian tumors, and 11 (9.4\%) had malignant ovarian tumors. Four malignant tumors were diagnosed in postmenopausal women and seven in premenopausal women.
The diagnosis based on grayscale imaging resulted in a correct specific diagnosis in $42 \%$ of patients and an incorrect specific diagnosis in $7 \%$ of cases. Doppler examination added to a correct specific diagnosis in $10.2 \%$, either by changing an incorrect specific diagnosis to a more correct one (five tumors) or by increasing the confidence with which a correct specific diagnosis was made. Doppler examination was misleading in one tumor.

Most of the adnexal masses were cystic (57\%), and $97 \%$ of cystic masses were benign. Sixty-five percent of cystic masses were simple unilocular cysts. Solid-cystic (complex) masses accounted for 35\%. Out of these, $78 \%$ were benign masses. Seven percent of the adnexal masses were solid, and $77.7 \%$ of the solid tumors were benign. Among the malignant adnexal masses, $18 \%$ were cystic, $63.3 \%$ were solidcystic, and $18 \%$ were solid. There was a statistically significant correlation between ovarian malignancy and the presence of a solid component (82\%), multiloculations (92\%), thickened walls and septae (82\%), papillary projections from the inner wall (73\%), and ascites (63\%), $p$ value $=0.000$ (Figure 1 ). The presence of dense echogenic contents of the cyst was found in cystadenomas and endometriomas. The characteristic homogenous carpet of low-level echoes was found in $78 \%$ of endometriomas. The results were promising but specificity was not high enough. We found sensitivity of $82 \%$ and specificity of $80 \%$. The positive predictive value was $30 \%$, and the negative predictive value was $98 \%$ (Table 1 ). The positive predictive value among postmenopausal women was $50 \%$.

Assessment of tumor vasculature by Doppler ultrasound revealed a mean PI of $1.6 \pm 0.4$ in benign masses vs. $0.7 \pm 0.1$ in malignant neoplasms. Mean RI values were $1.2 \pm 0.3$ and $0.4 \pm 0.1$ in benign and malignant masses, respectively. Considering the location of vessels, our results showed peripheral vascularity in $85 \%$ of benign masses, while in malignant neoplasms central and septal vascularization was more frequent (91\%). Figure 2 shows mean RI values at different locations in benign and malignant masses.

The other important parameter in the assessment of tumor blood vessels was their arrangement. Vessels were categorized as no vessel seen, regularly separated vessels, and randomly dispersed vessels. Among malignant tumors, 91\% exhibited randomly 
Table 1. Sensitivity, specificity, and predictive values for malignancy using the two modes of ultrasonography.

\begin{tabular}{lcccc}
\hline Mode of Ultrasound & Sensitivity & Specificity & $\begin{array}{c}\text { Positive Predictive } \\
\text { Value }\end{array}$ & $\begin{array}{c}\text { Negative Predictive } \\
\text { Value }\end{array}$ \\
\hline Grayscale & $82 \%$ & $80 \%$ & $30 \%$ & $98 \%$ \\
Color Doppler & $91 \%$ & $95 \%$ & $67 \%$ & $99 \%$ \\
\hline
\end{tabular}

distributed vasculature, compared to $22 \%$ of benign masses. Regularly separated vessels were more frequent among benign masses (59\% vs. 9\%). Eighteen percent of benign masses were avascular, while all the malignant tumors were highly vascular.

There was a group of benign lesions, which demonstrated a range of Doppler flow from high resistance to low resistance. These included dermoid cysts and endometriomas. Inflammatory masses were extremely well perfused, and vascular impedance varied from very low to very high (RI 0.4-2.3), giving a false-positive result of ovarian malignancy in two cases. By using color Doppler results for the prediction of malignancy, a high sensitivity of $91 \%$ and specificity of $95 \%$ were achieved. Positive predictive value was $67 \%$, and negative predictive value was $99 \%$ (Table 1). One false negative result was obtained in a borderline tumor, probably due to a lack of neovascularization in the early stage of malignant alteration.

Another major application of color Doppler sonography was in the diagnosis of ovarian torsion (7\%) and ectopic pregnancy (3\%). A high-velocity low-impedance Doppler signal was obtained from the area of developing placentation in cases of ectopic pregnancy. Torsion typically affects flow from both the ovarian artery and the adnexal branch of the uterine artery. Typically there was absent arterial flow within an enlarged ovary, which demonstrated irregular solid areas related to hemorrhage.

All the patients underwent surgery. Twenty percent of the patients had operative laparoscopy, $10 \%$ had laparoscopy followed by laparotomy, and 65\% underwent exploratory/staging laparotomy. According to histopathologic results of the malignant masses, serous cystadenocarcinomas were the most common (47\%), followed by mucinous cystade-

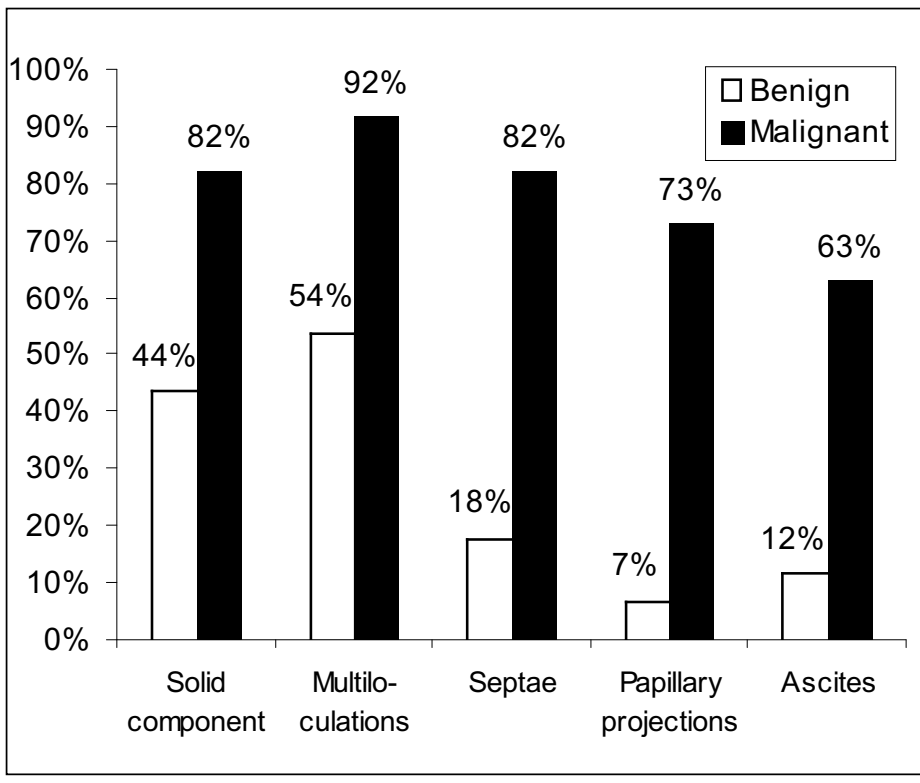

Figure 1. Grayscale echopatterns.

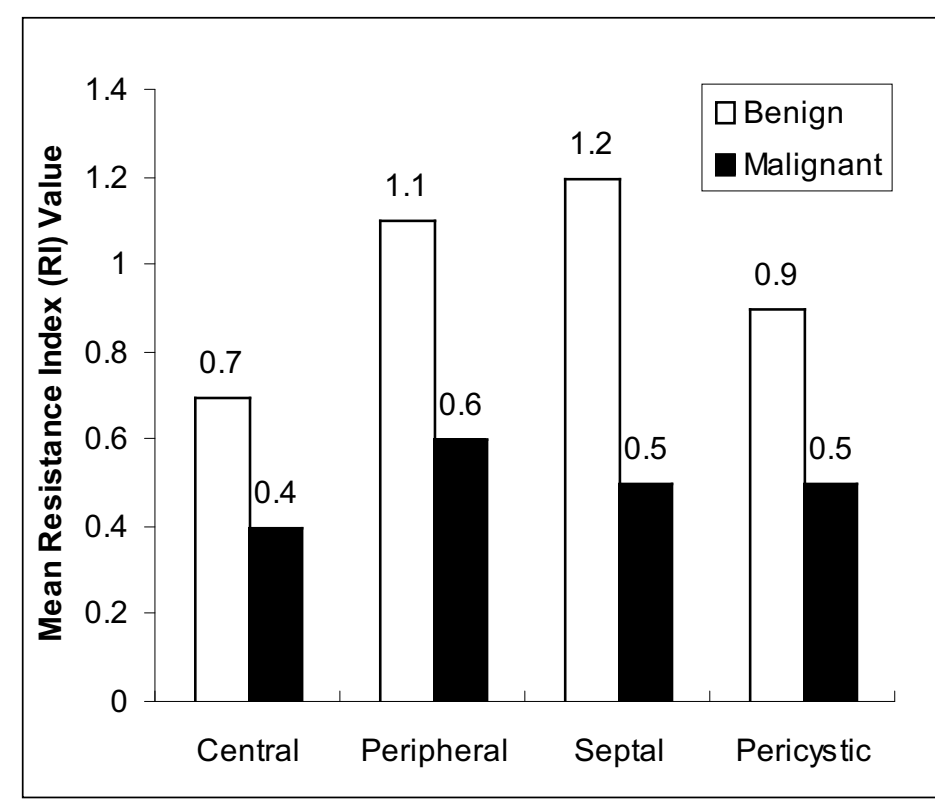

Figure 2. Mean resistance indices at different locations in benign and malignant lesions. 
nocarcinomas (27\%), endometriods (9\%), clear cell (9\%), and borderline tumors (9\%). Among benign masses, functional cysts were the most common (34\%), followed by cystadenomas (16\%), inflammatory masses $(15 \%)$, dermoid cysts $(13 \%)$, endometriomas (9\%), and paroophoron (7\%).

\section{Discussion}

The differential diagnosis of adnexal masses, including their benign and malignant nature, plays a major role in defining the appropriate surgical strategy, which in the case of malignancy allows a longer disease-free period and even a cure. Initially Meire et al examined the accuracy of grayscale ultrasound in delineating a malignant ovarian mass and found fixed septa, tumor size exceeding $5 \mathrm{~cm}$, and multiloculations ominous for ovarian malignancy. However only 16 (59\%) of 27 patients in their study with such findings were found to have ovarian cancer. ${ }^{26}$ In our study morphologic characteristics associated with a strong likelihood of malignancy on grayscale ultrasound were the presence of a solid component (82\%), multiloculations (92\%), and thickened septae. Buy et al reported a sensitivity of $88 \%$ and a specificity of $82 \%, 27$ while in another study, Stein et al reported the grayscale to have a sensitivity of $98 \%$ and a specificity of $62 \%$ for discerning malignant adnexal masses. 28 We found a sensitivity of $82 \%$ and a specificity of $80 \%$ in the present study, and in the subgroup of postmenopausal women, both sensitivity and specificity were higher, $90 \%$ and $84 \%$, respectively. An overall literature review reflects a high sensitivity and a lower specificity of grayscale in the diagnosis of benign masses; although recently, Troiano et al reported a sensitivity and specificity of $97 \%$, with no false-negative diagnoses of malignancy. ${ }^{29}$

Color Doppler ultrasound is a relatively new technique available to clinicians. Results of various studies have shown that increased vascularity of a mass, central location of the vessels (evidence of angiogenesis), and septal vascularization with low RI values are suggestive of malignancy. Advantages of this technique were demonstrated and used for the first time in gynecology by groups in Zagreb and London; both groups agree that this technique can detect ovarian cancer as early as International Federation of Gynecology and Obstetrics (FIGO) stage Ia disease. ${ }^{30} \mathrm{~A}$ high resistance index to flow can be used to exclude invasive primary ovarian cancer. ${ }^{31}$
The results of color Doppler sonography obtained in our study also support the use of Doppler sonography in the preoperative assessment of a pelvic mass. Both its sensitivity and specificity were higher than those of grayscale ultrasound, $91 \%$ vs. $82 \%$ and $95 \%$ vs. $80 \%$, respectively.

Mean RI for benign and malignant masses were 1.2 and 0.4, respectively. Mean PI values for benign and malignant masses were 1.6 and 0.7 , respectively. Fleischer and co-workers also described a significant difference between PI values of benign $(1.8 \pm 0.8)$ and malignant masses $(0.8 \pm 0.6) .21,32$ Hata and colleagues found low impedance blood flow in eight cases of ovarian cancer as well as four benign lesions. ${ }^{22}$ Bourne and associates reported seven cases of primary ovarian carcinoma. In six of these cases, areas of neovascularization and low PI values were found. A false-negative result was obtained from an early borderline serous cystadenocarcinoma. ${ }^{31}$

Although cutoff values for RI and PI are quite controversial, values used to indicate malignancy were $<1.0$ and $<0.5$, respectively, as most of the published data showed a better sensitivity and specificity using lower PI values (i.e. 0.5 and lower), and results of this study also support these values. Fleischer and co-workers observed a significant overlap at low PI values $(<1.0)$. Using a cutoff value of RI below 0.4 to indicate malignancy led to a falsenegative result in two malignant tumors with an RI of 0.6 and 0.5.21,32 In contrast, Weiner et al published a report with only one false-positive result with these cutoff values. ${ }^{23}$ We had one false-negative result with an RI of 0.7 , which was considered benign using a cutoff value of $<0.5$. A recent report from Timor-Tritsch demonstrated a mean PI value of 1.15 for benign and a value of 0.45 for malignant tumors. ${ }^{24}$ These values are lower than in previous papers. At the same time, Kawai and colleagues suggested a PI cutoff value of 1.25.25 Despite different opinions regarding cutoff values, all authors agree that recognition of angiogenesis as a reference point for malignant changes within the ovary has proved to be a highly sensitive parameter. Although cutoff values presented in different studies have been criticized, these thresholds are used for statistical analysis.

Numerous studies have reported encouraging evidence that there is an obvious difference between benign and malignant tumor vascularization, yet some overlap exists. ${ }^{33-34}$ This was also observed in the 
present study, and moderate vascularity was documented in benign lesions i.e. $47 \%$ of inflammatory masses, $28 \%$ of endometriomas, and $15 \%$ of dermoid cysts. Fleisher and colleagues suggested that this overlap could be reduced by considering the location of vessels. They consider central vascularization as a predictor of angiogenesis. ${ }^{21}$ Kurjak and coworkers found that randomly dispersed vascular arrangement was associated with high angiogenic activity in that particular area, suggesting malignancy. 35 All of these three parameters - RI, vessel location, and arrangement - formed the skeleton of the latest published color and pulsed Doppler scoring system. In this way a sensitivity of $97 \%$ and specificity of $100 \%$ has been reported. ${ }^{36}$ Our results agree with the findings of Fleischer and Kurjak. We found four times higher vascularity in malignant masses compared to benign tumors, and we found that $90 \%$ of malignant masses had evidence of angiogenesis. Eighty-five percent of benign adnexal masses had pericystic or peripheral vascularization. On the other hand, central (90\%) and septal vessels as well as vessels within papillary projections (60\%) were more frequent in malignant tumors.

Although the number of postmenopausal women in our study was small $(\mathrm{N}=14)$, both modes had a higher positive predictive value in these women. This can be explained by the fact that benign conditions having relatively similar echopatterns on grayscale and high vascularity on color Doppler ultrasound as malignant tissues, (i.e. inflammatory masses, endometriomas) are less common in postmenopausal women compared to the reproductive age group, thus reducing the overlap and resulting in fewer false-positive results.

\section{Conclusion}

Added specificity afforded by Doppler substantiates its use in selected cases in which grayscale scan is equivocal or nondiagnostic. However, still there is a spectrum of findings and an overlap in values seen in some benign conditions, and sonologists should beware of this overlap, minimizing the false diagnosis of benign lesions as malignant.

\section{References}

1. Morgante G, la Marca A, Ditto A, et al. Comparison of two malignancy risk indices based on serum CA125, ultrasound score and menopausal status in the diagnosis of ovarian masses. $\mathrm{Br} \mathrm{J}$ Obstet Gynaecol. 1999;106:524-7.

2. Gillis CR, Hole DJ, Still RM, et al. Medical audit, cancer registration, and survival in ovarian cancer. Lancet. 1991;337:611-2.

3. Finkler NJ, Benacerraf B, Lavin PT, et al. Comparison of CA 125, clinical impression, and ultrasound in the preoperative evaluation of ovarian masses. Obstet Gynecol. 1988;72:659-64.

4. Vasilev SA, Schlaerth JB, Campeau J, et al. Serum CA 125 levels in preoperative evaluation of pelvic masses. Obstet Gynecol. 1988;71:751-6.

5. Poveda A. Advanced ovarian cancer: update, remarks and conclusions on optimal therapy. Int J Gynecol Cancer. 2000;10(S1):570-60.

6. Nugent D, Salha O, Balen AH, et al. Ovarian neoplasia and subfertility treatments. Br J Obstet Gynaecol 1998;105:584-91.

7. McGuire V, Jesser CA, Whittemore AS. Survival among U.S. women with invasive epithelial ovarian cancer. Gynecol Oncol. 2002;84:399-403.

8. Bilgin T, Karabay A, Dolar E, et al. Peritoneal tuberculosis with pelvic abdominal mass, ascites and elevated CA 125 mimicking advanced ovarian carcinoma: a series of 10 cases. Int J Gynecol Cancer. 2001;11:290-4.

9. Bell R, Petticrew M, Sheldon T. The performance of screening tests for ovarian cancer: results of systematic review. Br J Obstet Gynaecol. 1998;105:1136-47. 10. Ahmad KK, Shaukat A, Khosa HL, et al. The role of ultrasound in diagnosis gynecologic/pelvic tumours. Ann King Edward Med Coll. 2001;7:319-23.

11. Nevin J, Denny L, Soeters R et al. Ultrasonography of pelvic masses. Br J Obstet Gynaecol. 1998;105:1379.

12. Valentin L. Comparison of Lerner score, Doppler ultrasound examination and their combination for discrimination between benign and malignant adnexal masses. Ultrasound Obstet Gynecol. 2000;15:143-7.

13. Tekay A, Jouppila P. Validity of pulsatility and resistance indices in classification of adnexal tumors with transvaginal color Doppler ultrasound. Ultrasound Obstet Gynecol. 1992;2:338-44.

14. Burns PN. Measuring volume flow with Doppler ultrasound - an old nut. Ultrasound Obstet Gynecol. 1992;2:238-41.

15. Fleischer AC, Rodgers WH, Kepple DM, et al. Color Doppler sonography of ovarian masses: a multipara- 
meter analysis. J Ultrasound Med. 1993;12:41-8. 16. Twickler DM, Forte TB, Santos-Ramos R, et al. The ovarian tumor index predicts risk of malignancy. Cancer. 1999;86:2280-90.

17. Alcázar JL, Errasti $\mathrm{T}$, Zornoza A, et al. Transvaginal color Doppler ultrasonography and CA125 in suspicious adnexal masses. Int J Gynaecol Obstet. 1999;66:255-61.

18. Leeners B, Funk A, Schild RL, et al. [Preoperative determination of the structure of pelvic tumors with color-coded Doppler ultrasound and conventional transvaginal ultrasound diagnosis]. Zentralbl Gynakol. 1998; 120:503-10.

19. Takac I. Analysis of blood flow in adnexal tumors by using color Doppler imaging and pulsed spectral analysis. Ultrasound Med Biol. 1998;24:1137-41.

20. Meire HB, Farrant P, Guha T. Distinction of benign from malignant ovarian cysts by ultrasound. Br J Obstet Gynaecol. 1978;85:893-9.

21. Fleischer AC, Rodgers $\mathrm{WH}$, Rao BK, et al. Assessment of ovarian tumor vascularity with transvaginal color Doppler sonography. J Ultrasound Med. 1991;10:563-8.

22. Hata K, Makihara K, Hata T, et al. Transvaginal color Doppler imaging for hemodynamic assessment of reproductive tract tumors. Int J Gynaecol Obstet. 1991;36:301-8.

23. Weiner Z, Thaler I, Beck D, et al. Differentiating malignant from benign ovarian tumors with transvaginal color flow imaging. Obstet Gynecol. 1992;79:159-62.

24. Timor-Tritsch LE, Lerner JP, Monteagudo A, et al. Transvaginal ultrasonographic characterization of ovarian masses by means of color flow-directed Doppler measurements and a morphologic scoring system. Am J Obstet Gynecol. 1993;168:909-13.

25. Kawai M, Kano T, Kikkawa F, et al. Transvaginal Doppler ultrasound with color flow imaging in the diagnosis of ovarian cancer. Obstet Gynecol. 1992;79:163-7.

26. Sassone AM, Timor-Tritsch IE, Artner A, et al. Transvaginal sonographic characterization of ovarian disease: evaluation of a new scoring system to predict ovarian malignancy. Obstet Gynecol. 1991;78:70-6.

27. Buy JN, Ghossain MA, Hugol D, et al. Characterization of adnexal mases: combination of color Doppler and conventional sonography compared with spectral Doppler analysis alone and conventional sonography alone. AJR Am J Roentgenol. 1996;166:385-93.

28. Stein SM, Laifer-Narin S, Johnson MB, et al. Differentiation of benign and malignant adnexal masses: relative value of gray-scale, color Doppler, and spectral sonography. AJR Am Roentgenol. 1995;164:381-6.

29. Troiano RN, Quendens-Case C, Taylor KJ. Correlation of findings on transvaginal sonography with serum CA 125 levels. AJR Am Roentgenol. 1997;168:1587-90.

30. Kurjak A, Zalud I, Jurkovic D, et al. Transvaginal color Doppler for the assessment of pelvic circulation. Acta Obstet Gynecol Scand. 68:131-5.

31. Bourne T, Campbell S, Steer C, et al. Transvaginal colour flow imaging: a possible new screening technique for ovarian cancer. BMJ.1989;299:1367-70.

32. Fleischer AC, Rodgers WH, Rao BK, et al. Transvaginal color Doppler sonography of ovarian masses with pathological correlation. Ultrasound Obstet Gynecol. 1991;1:275-8.

33. Kurjak A, Zalud I, Schulman H. Adnexal masses. In: Kurjak A, Editor Transvaginal color Doppler. Carnforth: Parthenon Publishing; 1990. pp. 93-101.

34. Kobal B, Rakar S, Ribic-Pucelj $M$, et al. Pretreatment evaluation of adnexal tumors predicting ovarian cancer. Int J Gynecol Cancer. 1999;9:4816.

35. Kusnetzoff D, Guochi D, Damonte C, et al. Differential diagnosis of pelvic masses: usefulness of CA125, transvaginal sonography and echo-Doppler. Int J Gynecol Cancer. 1998;8:315-21.

36. Kurjak A, Predavic A, Kupesic S, et al. Adnexal masses- malignant ovarian tumors. In: Kurjak A, ed. An atlas of transvaginal color Doppler: the current state of the art. Carnforth: Parthenon; 1994. pp. 291316. 